\title{
Angular Dependence of Energy Loss in Proton-Helium Collisions
}

\author{
G. Schiwietz and P. L. Grande* \\ Hahn-Meitner-Institut GmbH, Abteilung FD, Glienicker Strasse 100, 14109 Berlin, Federal Republic of Germany \\ C. Auth and H. Winter \\ Fachbereich Physik der Humboldt-Universität zu Berlin, Invalidenstrasse 110, 10115 Berlin, Federal Republic of Germany \\ A. Salin \\ Laboratoire des Collisions Atomiques, Centre National de la Recherche Scientifique \\ and Université Bordeaux I, 351 Cours de la Libération, 33405 Talence, France
}

(Received 12 April 1993)

\begin{abstract}
The energy loss of 50 to $250 \mathrm{keV}$ protons scattered under single-collision conditions from He atoms is investigated in terms of its dependence on the angle of scattering. At the higher projectile energies we observe an enhanced energy loss at scattering angles around $0.5 \mathrm{mrad}$. Such a behavior cannot be understood on the basis of two-body scattering models. Based on our theoretical studies we show that the combined effects of the screened target potential and of electronic transitions have to be considered for the energy loss of proton scattering in light gases.
\end{abstract}

PACS numbers: $34.50 . \mathrm{Bw}, 34.50 . \mathrm{Fa}, 61.80 . \mathrm{Mk}$

Recently energy-loss phenomena of fast ions in gases have gained increasing attention [1,2], where the stopping of the projectiles proceeds in a sequence of ion-atom collisions. Despite the relevance of atomic collisions for a description of energy dissipation in gases and also in solids, the understanding of energy loss in those elementary processes is far from being complete. For relatively high projectile velocities $v$, i.e., $v\rangle^{\prime} v_{e}$, the typical electron orbital velocities in the target atom, calculations in firstorder Born approximation are assumed to provide a good description [3-5]. However, for intermediate velocities $\left(v \approx v_{e}\right)$ one has to apply numerical solutions of the time-dependent Schrödinger equation that go beyond perturbative treatments [6,7]. In these (ab initio) calculations the mean energy loss of projectiles interacting with a single target atom is obtained as a function of impact parameter or scattering angle. Because of the apparent experimental difficulties no corresponding data for the stopping of light projectiles by light atoms have been reported so far.

In this Letter we present a theoretical as well as an experimental study on the energy loss of fast protons in single collisions with $\mathrm{He}$ atoms. The energy spectra for scattered protons are recorded with high energy and high angular resolution. Proton beams with energies from 50 to $250 \mathrm{keV}$ and energy spreads $\delta E / E \approx 5 \times 10^{-5}$ (absolute stability of energies 1 to $2 \mathrm{eV}$ per h) are collimated by a set of slits, collide in a gas cell under single-collision conditions with $\mathrm{He}$ atoms, and are scattered towards the entrance slit (width $\approx 30 \mu \mathrm{m}$ ) of an electrostatic analyzer made of cylindrical electrodes with $0.5 \mathrm{~m}$ radius. The overall energy resolution of the setup is $\delta E /$ $E<10^{-4}$, and the angular resolution is better than $\pm 0.15 \mathrm{mrad}$. The experiments have been performed at the Institute für Kernphysik (Münster) and a detailed description of the experimental procedures will be given elsewhere [8].

As a representative example we display in Fig. 1 an energy-loss spectrum from $200 \mathrm{keV}$ protons for a projectile scattering angle of $1 \mathrm{mrad}$. The dominant peak is due to elastic collisions and indicates an overall energy resolution of about $9 \mathrm{eV}$. The second peak at about $23 \mathrm{eV}$ corresponds mainly to single-excitation processes. Double excitation should lead to energy losses between 58 and 79 $\mathrm{eV}$ and a structure seems to be present at those energies. Also displayed in Fig. 1 are results of eikonal-AO calculations (thin solid line; atomic-orbital calculations, see below) where the thick solid line shows the results convoluted with the experimental energy resolution. The

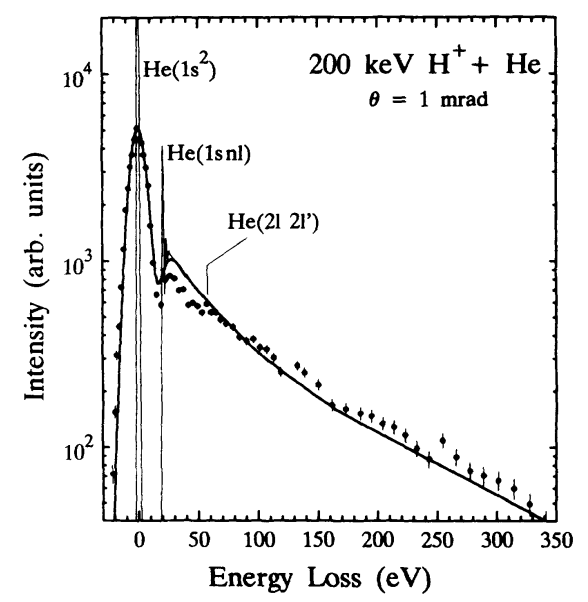

FIG. 1. Measured energy-loss spectrum for $200 \mathrm{keV} \mathrm{H}^{+}$incident on $\mathrm{He}$ at a projectile scattering angle of $1 \mathrm{mrad}$ in comparison with (three-body) eikonal-AO results, normalized to the integral of the experimental data, obtained from Eqs. (1) and (2) (thin solid line). Eikonal-AO results convoluted with a Gaussian distribution: thick solid line. 
theory reproduces the data very well. The slight differences may be due to effects of two-electron processes [9], since the theoretical results presented here are based on the independent-electron model. It is noted that the spectra taken at other scattering angles do not show additional structures.

In Fig. 2 we show experimental mean energy-loss data for 50 to $250 \mathrm{keV}$ protons on $\mathrm{He}$ as a function of projectile scattering angle. The data were obtained from an integration of the energy-loss spectra (see Fig. 1), where we estimate the resulting overall error in the integration and extrapolation methods to about $\pm 5 \%$. We note that the high energy resolution available here is a crucial prerequisite for this analysis of data. At projectile energies of 50 and $100 \mathrm{keV}$ the data sets show a monotonic increase of the mean energy loss with increasing scattering angle. For incident energies above $100 \mathrm{keV}$, however, we observe a peak structure in the mean energy loss at scattering angles around $0.5 \mathrm{mrad}$.

The peak around $0.5 \mathrm{mrad}$ can be related to the socalled binary process: If the projectile interacts with a free electron initially at rest, each final electron energy corresponds to a well-defined projectile-scattering angle. The binary process gives rise to a maximum in the differential ionization cross section for a given electron energy and therefore to a maximum in the mean energy transfer as a function of projectile scattering angle [10]. This maximum appears in our experiments only at incident energies above $100 \mathrm{keV}$. For lower energies however, two factors have to be taken into account: First, for a given ejected electron energy, the maximum is less apparent, and second the relative yield of low energy electrons (which give a smooth contribution around 0.5 mrad) is larger [10]. In summary, we can distinguish three different regimes with respect to the projectile scattering mechanism.

(i) At high incident energies the center of the energyloss peak corresponds mainly to electron-projectile (twobody) interactions with large transferred momenta. For ionizing collisions a shoulder was observed in the angular

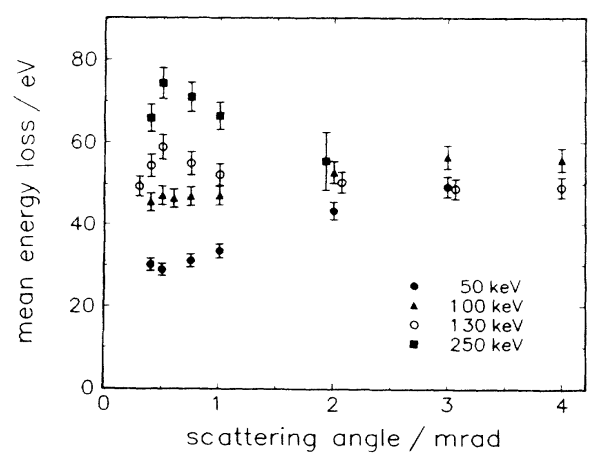

FIG. 2. Experimental mean electronic energy loss for 50 to $250 \mathrm{keV} \mathrm{H}^{+}$on $\mathrm{He}$ as a function of the $\mathrm{H}^{+}$scattering angle. dependence of single-ionization cross sections near 0.5 mrad at incident energies from 3 to $9 \mathrm{MeV}$ [11]. In another work [12] it was shown that this shoulder appears at incident energies above $400 \mathrm{keV}$. The energyloss peak investigated in the present work is much more pronounced and it is visible already at $130 \mathrm{keV}$, since both the ionization probability and the mean energy transfer per ionization event are quite high at $0.5 \mathrm{mrad}$ (65\% and $120 \mathrm{eV}$ at $200 \mathrm{keV})$. It should be noted, however, that the mean ejected-electron energies as well as the electron-energy distributions determined from our data differ significantly from the simplified two-body kinematics (only the kinematic low-energy branch of the energy loss was considered) on which the interpretation of Kamber et al. [11] is based. These deviations are due to the existence of two kinematic branches for small scattering angles, due to the initial velocity distribution, and due to three-body effects as a result of projectiletarget interactions and electron-target interactions.

(ii) At larger angles $(\Theta>3 \mathrm{mrad})$, the projectile scattering is due to the projectile-target-core interaction. The excitation and ionization probabilities are close to those for zero impact parameter and the energy loss is therefore nearly independent of the angle.

(iii) For intermediate scattering angles $(0.6 \mathrm{mrad}$ $<\Theta<3 \mathrm{mrad}$ ) as well as for small scattering angles $(\Theta<0.4 \mathrm{mrad})$ the projectile interacts simultaneously with the target nucleus and one of the electrons (threebody effects). For small angles the energy loss is dominated by excitation and emission of low energy electrons. For this case Dörner et al. [13] have observed three-body effects in measurements of the angular dependence of the (nuclear) energy transfer to $\mathrm{He}^{+}$ions. Another threebody effect leads to a maximum in the capture probability at high incident energies [14]. However, the influence of electron capture to our mean energy-loss data is of minor importance at energies above $100 \mathrm{keV}$.

The ion-atom collision investigated here is at least a three-body problem involving the projectile interaction with the active electron and with the target core. For incident energies above a few hundred $\mathrm{eV}$ and small projectile scattering angles, the solution to this problem can be considerably simplified by employing the eikonal method [15]. One first solves the time-dependent Schrödinger equation for rectilinear projectile trajectories (impactparameter method). The transition amplitude as a function of projectile scattering angle is then obtained from the amplitudes in the impact-parameter method through a Hankel transformation. The time-dependent Schrödinger equation is solved in this work by expanding the electronic wave function $\Phi_{e}(t)$ in terms of atomic orbitals $\varphi_{i}$ with coefficients $a_{i}(t)=\left\langle\varphi_{i} \mid \Phi_{e}(t)\right\rangle$ [6]. Thus, the Schrödinger equation is replaced by a set of coupled first-order differential equations. Neglecting the recoil motion of the target, the so-called coupled-channel equations can be written (in atomic units) as 


$$
i \frac{d}{d t} a_{i}(t, b)=\sum_{j} a_{j}(t, b) e^{i\left(E_{i}-E_{j}\right) t}\left\langle\varphi_{i}\left|V_{p}(\mathbf{r}-\mathbf{R}(t))\right| \varphi_{j}\right\rangle
$$

with orbital energies $E_{i}$ associated with the target wave function $\varphi_{i}$ and with the interaction potential $V_{p}$ between the projectile and the target electrons. The internuclear distance $\mathbf{R}=\mathbf{v} t+\mathbf{b}$ is given by straight-line trajectories of the atomic nuclei, where $\mathbf{b}$ and $\mathbf{v}$ are the impact parame- ter and projectile velocity, respectively.

In the present work, the four-body problem is reduced to a three-body problem by application of the independent-electron frozen-core model; i.e., the target is represented by a single electron evolving in the initial-state Hartree-Fock potential. The two-electron amplitude can then be obtained from the product of two single-electron amplitudes [16]. The differential scattering amplitude for a small projectile scattering angle $\boldsymbol{\theta}$, the eikonal approximation [15], reads

$$
f_{n_{1}, l_{1}, m_{1} ; n_{2}, l_{2}, m_{2}}(\Theta)=(i)^{m_{1}+m_{2}} K \int_{0}^{\infty} b d b\left[a_{n_{1}, l_{1}, m_{1}}(\infty, b) a_{n_{2}, l_{2}, m_{2}}(\infty, b) e^{-i \int d t Z_{p} Z_{l} / R}-\delta_{1,2 ; \mathrm{GS}}\right] J_{\left|m_{1}+m_{2}\right|}(K \Theta b)
$$

for a bare projectile with charge $Z_{p}$ and for a target nuclear charge $Z_{t}$. The principle quantum number is denoted $n_{i}$ and $l_{i}, m_{i}$ are quantum numbers associated with the angular momentum and angular momentum projection of the $i$ th electron. $\delta_{1,2 ; G S}$ is equal to 1 only when both electrons are in the ground state; otherwise it is zero and $J_{m}(z)$ are Bessel functions of integer order. $K$ is the momentum of the projectile in the laboratory frame. Equation (2) accounts for the combined influence of the target nuclear potential and individual electronic transitions on the projectile motion. Then, the mean electronic energy transfer can be directly computed from

$$
Q_{e}(\Theta)=\sum_{i}\left|f_{i}(\Theta)\right|^{2} \Delta E_{i} / \sum_{i}\left|f_{i}(\Theta)\right|^{2},
$$

since each two-electron state $i$ (specified by $n_{1}, l_{1}, m_{1}$; $\left.n_{2}, l_{2}, m_{2}\right)$ corresponds to a well-defined energy transfer $\Delta E_{i}=E_{i}-E_{0}\left(E_{0}\right.$ is the initial-state energy).

This procedure of predicting the energy loss vs the projectile scattering angle goes far beyond semiclassical models that use classical trajectories for the atomic nuclei and rely on model assumptions to convert impact parameter into projectile scattering angle. In fact it will be shown that semiclassical models underestimate the electronic energy transfer at projectile scattering angles of $0.5 \mathrm{mrad}$ by a factor of 2 . The theoretical results presented here (Figs. 1 and 3) are numerical solutions of Eqs. (1) and (2) and are accurate to within a numerical uncertainty of about $\pm 3 \%$. Because of the need for many partial waves at high continuum energies the corresponding energy transfers have been calculated using a basis set of about 250 gerade states (corresponding to about 350 one-electron eigenstates). The treatment of continuum states, computation time, and other details of the calculation are reported elsewhere [6].

Figure 3 displays the mean energy loss for protons incident on helium at 100 and $200 \mathrm{keV}$ as a function of the projectile scattering angle. The experimental results are identical to those presented in Fig. 2 (closed squares). It is noted that electron capture adds to the energy loss and becomes significant at lower incident energies. Thus, for $100 \mathrm{keV}$ we correct the experimental data for contributions due to electron capture (open squares). The capture probability is determined also in the experiments. The thick solid lines represent the eikonal-AO results as discussed above. Since we have performed large-basis-set AO calculations electron capture is automatically included here [6]. Hence, for $100 \mathrm{keV}$ the eikonal-AO results should be compared to the open squares. It is obvious that the agreement between the experimental and the theoretical results is not as good for $100 \mathrm{keV}$ as for 200 $\mathrm{keV}$. We assign this deviation to two-electron processes, which are not properly taken into account in any independent-electron treatment.

The dashed lines in Fig. 3 represent results that are also based on Eq. (1), with the same basis states as in the eikonal-AO calculations. The nuclear trajectories, however, are here obtained self-consistently through the classical Hamilton equations for an averaged heavy-particle Hamiltonian [6] that is computed from the timedependent electron density. This mean-field trajectory treatment goes beyond models that use predetermined

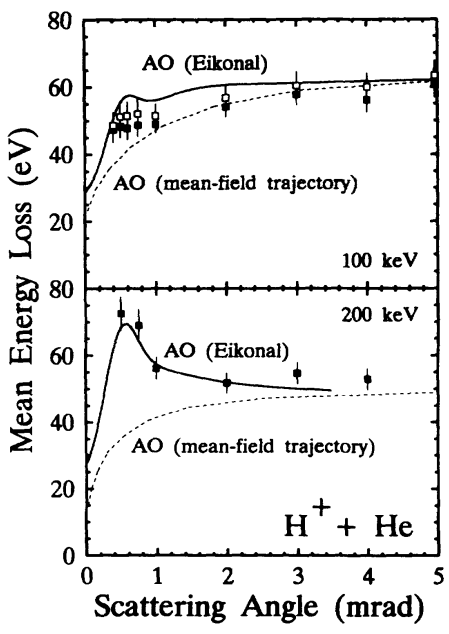

FIG. 3. Mean electronic energy loss for $\mathrm{H}^{+}$incident on $\mathrm{He}$ at 100 and $200 \mathrm{keV}$ vs projectile scattering angle. Closed squares with error bars: experimental results as in Fig. 2. Open squares with error bars: experimental results corrected for the electron capture contribution. Solid lines: (three-body) eikonal-AO results; dashed lines: (two-body) AO results for mean-field projectile trajectories. 
straight-line or hyperbolic trajectories, since target polarization is accounted for. Still, this present semiclassical approach does not give any structure in the energy loss above $100 \mathrm{keV}$. In fact, the average potential used in the definition of the average trajectory is unable to account for the kinematics of a violent ionizing collision in contrast to the eikonal method. As can be seen from Fig. 3, these violent collisions enhance the mean energy transfer by a factor of about 2 for this case. These deviations between mean-field and eikonal-AO results extend up to $\boldsymbol{\theta} \approx 3 \mathrm{mrad}$ and point to the importance of three-body effects.

In conclusion, we have measured the scattering-angle dependence of the electronic energy transfer for 50 to 250 $\mathrm{keV} \mathrm{H}^{+}+$He. A peak structure is observed in the mean energy loss as a function of the projectile scattering angle. Full calculations of the electronic stopping power are presented by considering each basic single-electron mechanism of the energy loss of protons penetrating a He gas target. The corresponding eikonal-AO results are the first theoretical energy-loss data that consistently account for the projectile-electron interaction. A good overall agreement of the results from this three-body theory (eikonal-AO) with experimental data is obtained. The remaining discrepancies of less than about $10 \%$ at intermediate energies are assigned to two-electron processes. Via a comparison between the eikonal-AO results and theoretical mean-field results (assuming two-body kinematics and thus a one-to-one correspondence between impact parameter and scattering angle) we are able to identify the origin of a peak structure in the scattering-angle dependence of the mean energy loss. This peak is due to violent projectile-electron collisions that lead to projectile deflections around $0.5 \mathrm{mrad}$.

Our calculations indicate that about $50 \%$ of the total proton energy loss at incident energies above $200 \mathrm{keV}$ is related to $0.5 \mathrm{mrad}$ scattering, in accord with the wellknown equipartition rule for the energy loss of fast particles. This correlation between angular scattering and energy loss is not taken into account in the transport theories for ions in matter $[17,18]$. The energy-loss peak may have special relevance for channeling/dechanneling of light charged particles and surface-scattering phenomena. At incident energies above $100 \mathrm{MeV}$, e.g., a single close encounter of a proton with an electron may lead to dechanneling in diamond even for a perfect lattice at $0 \mathrm{~K}$. Already at $\mathrm{MeV}$ energies one may expect a strong influence of the angular-scattering-energy-loss relation on the energy distribution of hyperchanneled particles [18]. Projectiles with exceptional high energy losses in the channel will preferentially be dechanneled and lose even more energy. Thus, the residual fraction of chan- neled ions corresponds to low energy losses.

One of us (A.S.) was partially supported by the Conseil Régional d'Aquitaine. P.L.G. acknowledges the support given by Hahn-Meitner Institut. Laboratoire des Collisions Atomiques is Unité propre de Recherches 260 du CNRS.

*Permanent address: Instituto de Física, Universidade Federal do Rio Grande do Sul, 91500 Porto Alegre, RS, Brazil.

[1] P. Bauer, F. Kastner, A. Arnau, A. Salin, P. D. Fainstein, V. H. Ponce, and P. M. Echenique, Phys. Rev. Lett. 69, $1137-1139$ (1992).

[2] R. Schuch, H. Schöne, P. D. Miller, H. F. Krause, P. F. Dittner, S. Datz, and R. E. Olson, Phys. Rev. Lett. 60, 925 (1988).

[3] J. T. Park, J. E. Aldag, J. M. George, J. L. Peacher, and J. H. McGuire, Phys. Rev. A 15, 508 (1977).

[4] A. Dalgarno and G. W. Griffing, Proc. R. Soc. London A 232, 423 (1955).

[5] L. L. Balashova, N. M. Kabachnik, and V. N. Kondratev, Phys. Status Solidi (b) 161, 113 (1990); N. M. Kabachnik, V. N. Kondratev, and O. V. Chumanova, Phys. Status Solidi (b) 145, 103 (1988).

[6] G. Schiwietz, Phys. Rev. A 42, 296-306 (1990); G. Schiwietz and P. L. Grande, Nucl. Instrum. Methods Phys. Res., Sect. B 69, 10-17 (1992); P. L. Grande and G. Schiwietz, Phys. Rev. A 47, 1119-1122 (1993).

[7] P. D. Fainstein, V. H. Ponce, and A. E. Martinez, Phys. Rev. A 47, 3055 (1993).

[8] C. Auth and H. Winter, Phys. Lett. A 176, 109-112 (1993); Nucl. Instrum. Methods (to be published).

[9] B. Skogvall and G. Schiwietz, Phys. Rev. Lett. 65, 3265 (1990); Phys. Rev. A 46, 5687 (1992).

[10] A. Salin, J. Phys. B 22, 3901-3914 (1989).

[11] E. Y. Kamber, C. L. Cocke, S. Cheng, and S. L. Varghese, Phys. Rev. Lett. 60, 2026 (1988).

[12] F. G. Kristensen and E. Horsdal-Pedersen, J. Phys. B 23, 4129 (1990).

[13] R. Dörner, J. Ullrich, H. Schmidt-Böcking, and R. E. Olson, Phys. Rev. Lett. 63, 147 (1989).

[14] E. Horsdal-Pedersen, C. L. Cocke, and M. Stockli, Phys. Rev. Lett. 50, 1910-1913 (1983).

[15] L. Wilets and S. J. Wallace, Phys. Rev. 169, 84 (1968); R. McCarroll and A. Salin, J. Phys. B 1, 163 (1968); M. R. C. McDowell and J. P. Coleman, Introduction to the Theory of Ion-Atom Collisions (North-Holland, Amsterdam, 1970).

[16] H. J. Lüdde and R. M. Dreizler, J. Phys. B 18, 107 (1985).

[17] J. P. Biersack, Nucl. Instrum. Methods Phys. Res., Sect. B 19, 32 (1987).

[18] D. S. Gemmell, Rev. Mod. Phys. 46, 129 (1974). 